\title{
Rôles et caractéristiques morphologiques du ver de terre Eudrilus eugeniae (synthèse bibliographique)
}

\author{
Patrick Byambas ${ }^{(1,3)}$, Aboulkacem Lemtiri ${ }^{(2)}$, Jean Luc Hornick ${ }^{(1)}$, \\ Toussaint Bengone Ndong ${ }^{(3)}$, Frédéric Francis ${ }^{(2)}$
}

\author{
(1) Université de Liège. Faculté de Médecine vétérinaire. Département des Productions animales. Boulevard de Colonster, 20 \\ bât. 43. BE-4000 Liège (Belgique).E-mail : jlhornick@ulg.ac.be \\ (2) Université de Liège - Gembloux Agro-Bio Tech. Entomologie fonctionnelle et évolutive. Passage des Déportés, 2. BE- \\ 5030 Gembloux (Belgique). \\ (3) Centre National de la Recherche Scientifique et Technologique (CENAREST). Institut de Recherches Agronomiques et \\ Forestières (IRAF). Trois quartiers. BP 2246. Libreville (Gabon).
}

Reçu le 13 mars 2016, accepté le 14 mars 2017.

Cet article est distribué suivant les termes et les conditions de la licence CC-BY (http://creativecommons.org/licenses/by/4.0/ deed.fr)

Introduction. Eudrilus eugeniae est un ver épigé d'origine africaine. Il est principalement rencontré dans les pays tropicaux et subtropicaux, et est utilisé dans les processus de lombriculture et de lombricompostage.

Littérature. Le développement de l'utilisation et de l'exploitation du ver nécessite un approfondissement des connaissances s'y rapportant pour optimiser la production des vers et du lombricompost. Cette synthèse bibliographique a été initiée pour faciliter la valorisation agronomique de l'espèce au Gabon et améliorer les connaissances actuelles sur E. eugeniae. Elle pourrait faciliter l'élaboration d'une clé d'identification spécifique à E. eugeniae, à l'instar de celles qui existent pour d'autres espèces.

Conclusions. La synthèse des connaissances disponibles sur le ver de terre E. eugeniae devrait faciliter son exploitation et sa valorisation dans les pays comme le Gabon où l'étude de ce ver de terre est récente.

Mots-clés. Eudrilus, vermicompostage, morphologie, vermiculture, Gabon.

Roles and morphological characteristics of the earthworm Eudrilus eugeniae. A review

Introduction. Eudrilus eugeniae is an aboveground African worm mainly found in tropical and subtropical countries and is used in the process of vermiculture and vermicomposting.

Literature. The development of the production of this earthworm species requires a deepening knowledge of the species. This review aimed to facilitate the agricultural recovery of the worm in Gabon and improve the current knowledge on E. eugeniae. Finally, the development of a specific identification key for E. eugeniae is recommended.

Conclusions. Further investigation regarding the geographical distribution of this worm in the equatorial zone, where it was recently described, must also be considered. This review of the available knowledge on the earthworm E. eugeniae should facilitate its use and its valorization in countries such as Gabon, where knowledge of this worm is recent.

Keywords. Eudrilus, vermicomposting, morphology, vermiculture, Gabon.

\section{INTRODUCTION}

Eudrilus eugeniae (Kinberg, 1867) est une espèce de ver de terre qui appartient à la famille des Eudrilidae (Ansari \& Saywack, 2011). C'est un invertébré qui appartient à l'Embranchement des Annelides, à la classe des Oligochètes et à l'ordre des Haplotaxidae qui regroupe des organismes principalement terrestres et vivant dans le sol (Ansari \& Saywack, 2011). Plusieurs études ont classé les vers de terre en trois catégories et en cinq familles à travers le monde. Les trois groupes écologiques qui regroupent les vers de terre sont :
- les vers épigés qui vivent en surface dans les amas de matières organiques et creusent peu ou pas de galeries dans le sol. Eudrilus eugeniae fait partie de ce groupe (Pérès et al., 2011) ;

- les vers anéciques occupent la couche supérieure du sol autour de $25 \mathrm{~cm}$, ils creusent des galeries verticales dont la longueur peut atteindre plusieurs mètres (Morin, 1999) ;

- les vers endogés vivent dans les couches plus profondes et creusent des galeries horizontales (Pérès et al., 2011). 
Les vers de terre représentent jusqu'à $70 \%$ de la biomasse du sol (Zirbes et al., 2011). Cinq mille espèces ont déjà été décrites à travers le monde, mais de nombreuses restent à découvrir et principalement dans les zones tropicales (Brown et al., 2013). Les vers de terre jouent un rôle majeur dans la dynamique des nutriments du sol, en modifiant leurs propriétés physiques, chimiques et biologiques (Kooch \& Jalilvand, 2008). Ils sont présents dans la majorité des écosystèmes terrestres, principalement en régions tempérées et tropicales. Eudrilus eugeniae joue un rôle économique relativement important en aviculture (Francis et al., 2003) et dans la pêche sportive (Morin, 1999). Il est souvent utilisé dans le processus de vermicompostage dans les pays tropicaux et subtropicaux. Il présente un intérêt pour la valorisation des déchets organiques dans ces zones (Vijaya et al., 2012) et se caractérise par un corps cylindrique allongé constitué d'anneaux successifs (Pérès et al., 2011).

Eudrilus eugeniae a été répertorié en Afrique. C'est un ver qui sort généralement la nuit d'où le nom de ver nocturne africain (Brown et al., 2013). L'intérêt scientifique pour ce ver a connu une croissance exponentielle cette dernière décennie. Ainsi, plusieurs études lui ont été consacrées pour décrire l'anatomie interne (Butt \& Grigoropoulou, 2010), le cycle de reproduction (Parthasarathi, 2007 ; Sivasankari et al., 2013), les paramètres de croissance et de reproduction dans différents substrats (Coulibaly \& Zoro Bi, 2010) et la description des organes de reproduction (Vijaya et al., 2012). Ces études récentes facilitent les processus d'identification d'E. eugeniae, bien qu'il n'existe toujours pas de clé d'identification spécifique à ce ver de terre. Eudrilus eugeniae a été rencontré au Ghana, au Nigéria, au Cameroun et au Gabon (Oboh et al., 2007 ; James et Divina, 2012). La lombriculture d'E. eugeniae a été réalisée dans ces pays, excepté au Gabon (James \& Divina, 2012).

Malgré les connaissances actuelles, son rôle économique et agronomique reste mal connu dans les pays où il a été répertorié. L'intérêt de cette synthèse bibliographique sur E. eugeniae est de mieux relever ses caractéristiques physiques, son rôle dans les écosystèmes terrestres, les productions animales et la protection de l'environnement. Elle vise à faciliter aussi son identification et sa valorisation dans les pays comme le Gabon, où il est encore peu connu.

\section{BIOLOGIE ET ANATOMIE D'EUDRILUS EUGENIAE}

\subsection{Origine et distribution}

Eudrilus eugeniae a été répertorié dans plusieurs pays africains (Oboh et al., 2007 ; James \& Divina, 2012). À la différence d'autres espèces de ver de terre que l'on peut trouver dans différentes parties du globe, le ver nocturne africain est principalement présent dans les sols tropicaux (Ansari \& Saywack, 2011). Il est absent dans les déserts, les zones continuellement enneigées ou glaciales et les montagnes (Ansari \& Saywack, 2011).

\subsection{Morphologie d'Eudrilus eugeniae}

La morphologie externe d'E.eugeniae adulte (Tableau 1) est caractérisée par un corps allongé et recouvert d'anneaux successifs appelés segments (Figure 1). Il peut atteindre un poids maximal de $4 \mathrm{~g}$ à l'âge adulte (Reinecke et al., 1992 ; Ansari \& Saywack, 2010).

Le peristomium est le premier segment qui entoure la cavité buccale; sur celui-ci se trouve une bosse ou un lobe appelé prostomium dont la forme est un des éléments qui permettent de déterminer l'espèce de ver de terre (Crow, 2012). Les deux premiers segments ne possèdent pas de soies. Ces dernières sont des poils très fins dont le nombre sur les segments varie selon le segment du corps. Eudrilus eugeniae en possède huit, disposées en quatre paires en position ventro-latérale. Les soies interviennent dans la locomotion et la

Table 1. Caractères morphologiques externes d'Eudrilus eugeniae (d'après Ansari \& Saywack, 2011 ; Vijaya et al., 2012) - External morphological characteristics of Eudrilus eugeniae (according to Ansari \& Saywack, 2011; Vijaya et al., 2012).

\begin{tabular}{ll}
\hline Caractères morphologiques & Eudrilus eugeniae \\
\hline Couleur du corps & $\begin{array}{l}\text { Avant : rouge foncé } \\
\text { Arrière : bleu violet }\end{array}$ \\
\hline Forme du corps & Cylindrique \\
Longueur du corps & 130 à $150 \mathrm{~mm}$ \\
Nombre de segments du corps & 145 à 207 \\
Forme des soies du corps & Sigmoïde \\
\hline Forme du prostomium & Épilobique ouvert \\
Soies du corps & Séparés \\
Longueur des soies & 0,42 mm \\
\hline Forme du clitellum & annulaire \\
Longueur du clitellum & 5,3 à 5,5 mm \\
Nombre de segments du clitellum & 6 à 8 \\
Début du clitellum à partir de la tête & $13^{\mathrm{e}}$ ou $14^{\mathrm{e}}$ segment \\
Position du pore génital mâle & Segment 17 \\
Position du pore génital femelle & Segment 14 \\
Nombre de pore génital mâle & 1 paire \\
Nombre de pore génital femelle & 1 seul \\
\hline
\end{tabular}




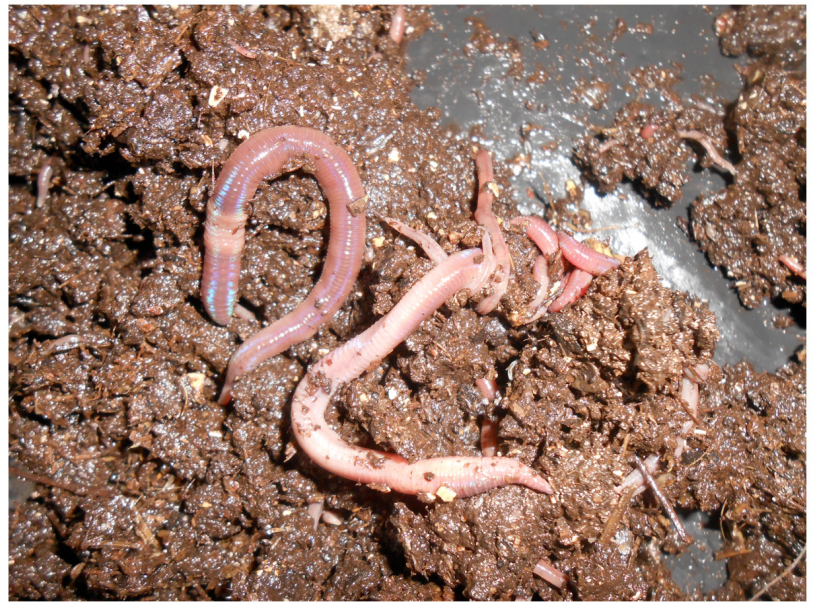

Figure 1. Spécimen de Eudrilus eugeniae dans du vermicompost - Specimen of Eudrilus eugeniae in vermicompost.

copulation (Crow, 2012). Le clitellum est un renflement qui indique la maturité sexuelle et apparait sur la partie antérieure du ver de terre. Il permet la production des cocons. Sur le dernier segment du corps, appelé pygidium ou anus, il n'y a pas de soies (Sivasankari et al., 2013).

\subsection{Anatomie d'Eudrilus eugeniae}

La disposition des organes internes du ver de terre révèle que la quasi-totalité des organes vitaux sont regroupés dans la partie antérieure de l'organisme, qui part de la tête au dernier segment du clitellum (Figure 2). La zone post-clitellaire débute après le clitellum et se termine à l'anus. Elle n'héberge qu'une partie de l'intestin (Yesudhason et al., 2013).

Une coupe transversale du ver de terre permet de distinguer l'épiderme externe ; un réseau de muscles circulaires et de muscles longitudinaux, séparés par un fin réseau de tissus nerveux; une membrane péritoine qui permet de délimiter la cavité cœlomique.

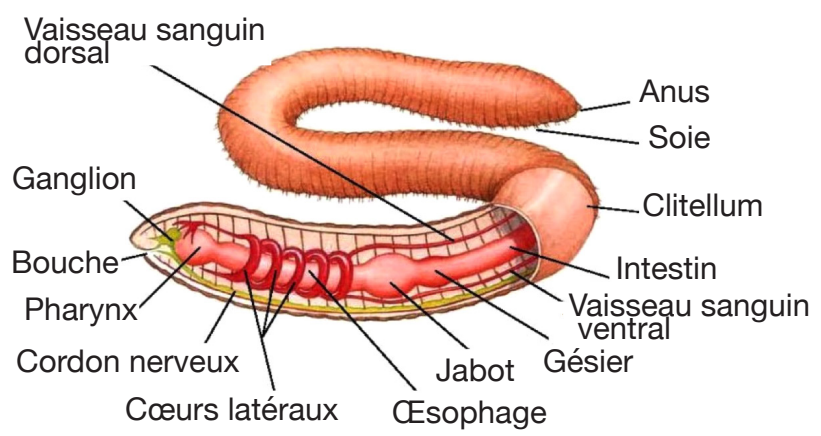

Figure 2. Anatomie interne d'Eudrilus eugeniae - Internal anatomy of Eudrilus eugeniae (www.infovisual.info).
L'épiderme externe est couvert d'une cuticule non cellulaire, fine et transparente qui couvre l'ensemble du corps. La cuticule réfléchit la lumière, mais elle permet aussi de faire ressortir les couleurs des couches sous cutanées, en laissant traverser la lumière (Siegrist, 2011).

Les vaisseaux dorsaux et ventraux se situent au-dessus et en dessous de l'intestin. Le cordon nerveux, qui relie les vaisseaux ventraux aux muscles longitudinaux, et la métanéphridie, qui est un organe excréteur métamérisé, s'ouvrent dans la cavité cœlomique. Ils sont situés dans la partie ventrale (Yesudhason et al., 2013).

Le tractus digestif (Figure 2) commence par la cavité buccale, qui absorbe les aliments par succion. Il comprend successivement le pharynx, l'œsophage, le jabot, le gésier, puis l'intestin qui se termine au pygidium. Plusieurs pseudo-cœurs entourent la zone de l'œsophage (Crow, 2012).

Le système de reproduction est composé de deux paires de testicules sous forme de lobe au niveau des cloisons des segments 10 et 11 , et celui des segments 11 et 12. Deux paires de larges vésicules séminales sont situées sur les segments 11 et 12 , ainsi que la glande de la prostate (euprostate) qui a la forme d'un doigt. Chez le ver E. eugeniae, les ovaires sont situés dans le $13^{\mathrm{e}}$ segment, dans la cavité cœlomique sous le canal alimentaire (Vijaya et al., 2012).

\subsection{Cycle de vie}

Le ver de terre E.eugeniae a un cycle de vie variant entre 50 et 70 jours (Reinecke et al., 1992). Le développement du clitellum commence entre 35 et 45 jours et la maturité sexuelle est atteinte entre 40 et 49 jours (Sivasankari et al., 2013). À la maturité sexuelle, les vers de terre s'accouplent et la formation du cocon intervient après 24 h (Sivasankari et al., 2013). Eudrilus eugeniae est un organisme hermaphrodite qui a besoin d'un partenaire pour se reproduire. Ils juxtaposent leurs organes de reproduction (Figure 3) en se positionnant tête-bêche (Morin, 2004). Le ver de terre peut aussi s'auto-coupler lorsqu'il est isolé ou pratiquer la parthénogénèse (Fernandez et al., 2011). Le

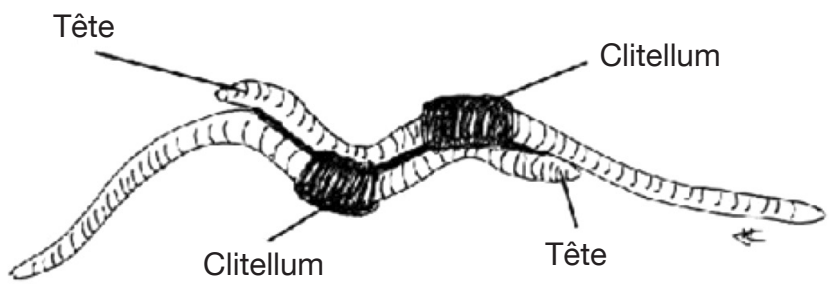

Figure 3. Position de reproduction des vers de terre Reproducing position of earthworms (Morin, 2004). 
clitellum permet aux partenaires de rester collés l'un à l'autre. Ils échangent leurs semences males et sécrètent de petits cocons via le clitellum (Morin, 2004).

Une semaine après l'accouplement, la production des cocons commence. La production peut atteindre 1,3 cocon par jour (Sivasankari et al., 2013). Le cocon produit a une couleur gris clair. Il durcit rapidement, puis devient gris orangé (Figure 4) et gris foncé juste avant l'éclosion. La longueur d'un cocon varie de 4,3 à $7,8 \mathrm{~mm}$, le diamètre varie entre 2,1 et $4 \mathrm{~mm}$ et le poids moyen est d'environ $17 \mathrm{mg}$ (Sivasankari et al., 2013). La période d'incubation des cocons varie de 12 à 16 jours avec un taux d'éclosion de 75 à $80 \%$ (Morin, 2004). Chaque cocon donne naissance à des juvéniles dont le nombre varie entre 1 et 4 par cocon (Dominguez \& Edwards, 2010).

Après un jour, le juvénile prend la forme du ver de terre adulte. Chez E. eugeniae, le temps entre la production du cocon et la maturité sexuelle dure $47 \pm 3$ jours à $25^{\circ} \mathrm{C}$ (Sivasankari et al., 2013).

En régions tropicales, dans des conditions favorables (températures $27^{\circ} \mathrm{C}$, humidité $75 \%$ ), un substrat adapté (matière organique animale et végétale, en décomposition) (Tahir \& Hamid, 2012), une population de ver de terre du type Eudrilus sp. croît rapidement et le juvénile peut atteindre le stade de maturité sexuelle en $35 \pm 3$ jours après l'éclosion (Sivasankari et al., 2013).

\section{TECHNIQUE DE LOMBRICULTURE}

La lombriculture ou vermiculture est le processus de production des vers de terre. Suivant l'espèce utilisée,

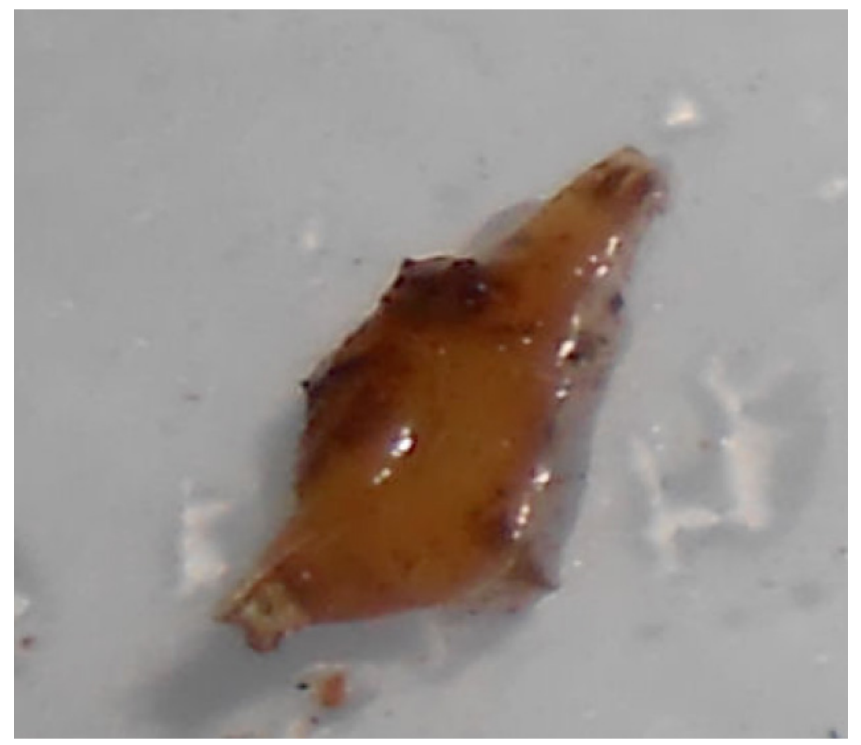

Figure 4. Cocon de l'espèce Eudrilus eugeniae - Cocoon of the species Eudrilus eugeniae. la densité, la température, l'épaisseur du substrat peuvent varier fortement et affecter la croissance et la reproduction des vers de terre (Chaoui, 2010). Les principales espèces utilisées en lombricompostage suivant le climat et la région sont Eisenia fetida, Eisenia andrei, Perionyx excavatus et Eudrilus eugeniae (Francis et al., 2003).

\subsection{Introduction des vers de terre}

Les vers de terre peuvent être issus d'un élevage d'E. eugeniae ou alors être prélevés par creusage et tri manuel dans les parties superficielles $(15 \mathrm{~cm}$ de profondeur) de l'horizon d'un sol abritant l'espèce recherchée. Les vers de terre devront tous être prélevés sur la même parcelle de terre afin de réduire la variation du biotype (Vijaya et al., 2012). Seuls les vers de terre matures sont sélectionnés pour la lombriculture. D'autres méthodes de prélèvement existent, telle que la placette de $1 \mathrm{~m}^{2}$ utilisant de la moutarde diluée (150 g.10 l' $^{-1}$ d'eau) en arrosage sur le sol ou l'arrosage d'une solution de formol sur la surface de prélèvement des vers de terre (Cluzeau, 2013).

\subsection{Mise en place de l'élevage}

La technique de lombriculture en zone tropicale se fait selon la méthode décrite par Francis et al. (2003) et Tahir \& Hamid (2012). Le bac plastique utilisé sera perforé dans le fond et sur le couvercle. Un bac supplémentaire devra permettre de recueillir le surplus d'eau en dessous du bac d'élevage. Les parois des bacs devront être peintes en noir pour maintenir les vers de terre dans l'obscurité (Tahir \& Hamid, 2012). Dans le bac, les différentes matières organiques devront êtres mélangées dans des proportions permettant d'avoir un rapport $\mathrm{C} / \mathrm{N}$ de 30 (Bruyeer \& Simus, 2012). Dans une exploitation où l'espace est plus grand, la lombriculture peut se faire au sol avec des tas de $1 \mathrm{~m}$ de haut et $3 \mathrm{~m}^{2}$ de surface (Francis et al., 2003). La lombriculture peut se pratiquer dans des bacs plastiques (Tahir \& Hamid, 2012), des constructions en terre cuite ou en bois (Francis et al., 2003), sur un film plastique sous serre (Morin, 1999) en fonction du milieu, de la surface disponible et des matériaux accessibles au producteur.

Pour faciliter son développement, sa reproduction et sa croissance, E. eugeniae a besoin d'une température variant entre 25 et $28^{\circ} \mathrm{C}$ ainsi qu'un $\mathrm{pH}$ de 6,4 à 7,0 (Tahir \& Hamid, 2012). L'humidité doit être maintenue entre 70 et $85 \%$ par arrosage chaque fois que c'est nécessaire (Dominguez \& Edwards, 2010). Quand on ne peut pas utiliser de l'eau distillée (Coulibaly et al., 2011), l'eau de pluie est préférable à l'eau du robinet qui peut potentiellement contenir du chlore (Lowe \& Butt, 2005). L'introduction des vers de terre n'intervient qu'après une période de précompostage 
de 14 jours (Nayak \& Sahu, 2013) pour permettre l'évacuation d'éventuels gaz volatils qui peuvent être toxiques pour le ver de terre (Coulibaly et al., 2016). La densité recommandée est de $1,6 \mathrm{~kg} \cdot \mathrm{m}^{-2}$ de substrat (Francis et al., 2003), ou 7 vers de terre adultes par litre (Deka et al., 2011). Le substrat d'élevage peut être constitué de différentes matières organiques disponibles dans la zone d'élevage (Morin, 2004). Le papier peut être utilisé pour constituer le substrat de la lombricompostière (Jesikha, 2015). Toutefois, l'usage du papier glacé ainsi que les papiers imprimés avec des encres de couleurs sont à éviter car elles peuvent être toxiques pour le ver de terre (Morin, 2004).

\subsection{Alimentation du ver de terre E. eugeniae}

Eudrilus eugeniae, comme la majorité des vers de terre, se nourrit de la matière organique présente en surface du sol (Sivasankari et al., 2013). Ils peuvent se nourrir de déjections d'autres organismes du sol. Cependant, un aliment adéquat est composé de graines moulues ou de sous-produits de céréales tels que le germe de blé, le son de blé, le maïs et les flocons d'avoine (Dominguez \& Edwards, 2010). En élevage, l'aliment peut être apporté aux vers de terre une à deux fois par semaine. La quantité apportée doit être égale à la biomasse des vers présents (Chaoui, 2010). Schuch et al. (2010) rapportent que les micro-organismes (bactéries) constituent une partie du régime alimentaire des lombrics. La nourriture est aspirée par la bouche et le pharynx musculeux, puis stockée dans le jabot, ensuite broyée dans le gésier, en particules très fines. L'absorption des éléments nutritifs s'effectue au niveau de l'intestin et l'autre partie non absorbée continue vers l'anus (Crow, 2012). La matière organique se minéralise dans l'intestin en forme absorbable par les plantes. Dans ce processus, les bactéries sont souvent éliminées car elles ne survivent pas dans l'intestin qui est peu oxygéné (Chaoui, 2010 ; Schuch et al., 2010).

\subsection{Récolte des vers de terre}

Eudrilus eugeniae peut se développer très rapidement en zone tropicale. Au moment de la récolte, le mélange de lombricompost est épandu sur un film plastique exposé au soleil, puis les vers sont triés à la mains (Francis et al., 2003). Suivant les surfaces utilisées, le niveau de production peut varier. Au Canada, des élevages produisent jusqu'à 1000000 de vers de terre sur $1600 \mathrm{~m}^{3}$ (Morin, 1999). Au sud Vietnam, 24 à $60 \mathrm{~kg}$ de vers de terre ont été produits chaque mois sur 6 à $15 \mathrm{~m}^{2}$, pour un cout de 27,5 EUR $\cdot \mathrm{m}^{-3}$ par unité de lombricompostage (Francis et al., 2003). Rajendran et al. (2008) rapportent que pour produire $70 \mathrm{t}$ de vers de terre par an, $1500 \mathrm{~m}^{2}$ de surface et 6 ouvriers sont nécessaires en Inde.

\section{RÔLES DU VER DE TERRE}

Le ver de terre a vu son importance grandir grâce aux différents rôles qu'il joue dans l'économie, l'environnement, l'agronomie et la zootechnie, notamment. En effet, les multiples usages du ver de terre font de lui un atout important pour la gestion des déchets organiques, la fertilisation des terres agricoles et l'alimentation animale (Coulibaly et al., 2014 ; Temgoua et al., 2014).

Le lombricompostage permet aux vers de terre, tels qu'Eisenia fetida, E. eugeniae et Perionyx excavatus, de décomposer des déchets organiques en éléments nutritifs assimilables par les plantes grâce la libération des éléments simples comme l'azote, le phosphore, le potassium et le calcium. Ce procédé permet aussi à la plante de disposer de substances biologiques actives telles que les régulateurs de croissance des plantes (Coulibaly et al., 2014). Le vermicompostage est une alternative à la fertilisation chimique des terres dans les pays tropicaux (Karmakar et al., 2012). Cette technique a permis à des exploitants agricoles d'avoir des revenus supplémentaires avec la commercialisation des vers de terre (Misra et al., 2005).

Les déchets organiques sont des sources alimentaires pour les vers de terre qui, à leur tour, représentent une source non négligeable de protéine pour l'alimentation animale (Munroe, 2006). Eudrilus eugeniae figure parmi les espèces de vers de terre les plus utilisées pour le vermicompostage.

\subsection{Importance économique}

Le ver de terre joue un rôle économique dans différents pays où il est exploité pour des besoins divers. Aux Philippines et en Inde, pour réduire leurs charges d'exploitations, les producteurs agricoles utilisent le vermicompost pour baisser les couts de la fertilisation. Dans ces pays, le fumier d'élevage, utilisé comme substrat pour le vermicompostage, est devenu un produit commercial assez onéreux pour les agriculteurs (FAO, 2004). Et le surplus de vers de terre produit est vendu à d'autres agriculteurs comme source d'aliment pour l'aviculture, la pisciculture ou pour ensemencer d'autres lombricompostages (Misra et al., 2005), ce qui est une source supplémentaire d'entrée de devises pour l'exploitation. Adorada (2007) a montré que des paysans philippins ont pu générer des bénéfices relativement importants avec la commercialisation des vers de terre et du vermicompost.

Les vers de terre sont aussi utilisés par les pêcheurs, les éleveurs débutants, les laboratoires expérimentaux (Dominguez \& Edwards, 2010). Au Canada, l'utilisation des vers de terre pour la pêche sportive a permis de générer des revenus annuels de près de 10 à 12 millions USD pour l'industrie de la lombriculture 
(Dominguez \& Edwards, 2010). Eudrilus eugeniae est l'un des vers de terre dont l'importance économique est reconnue (Loongyai et al., 2011). Sa valeur économique a pris de l'importance avec son utilisation comme appât pour la pêche sportive (Moraes et al., 2012).

\subsection{Importance environnementale}

La forte industrialisation et la croissance démographique ont un impact réel sur l'environnement, notamment par la production importante des déchets organiques qui posent des défis majeurs de gestion dans les pays tropicaux, en général et ceux en voie de développement, en particulier (Temgoua et al., 2014). C'est pour répondre à ces défis que plusieurs voies sont explorées, dont celle du traitement organique des déchets à travers le vermicompostage (Yadav \& Garg, 2011). Plusieurs espèces, dont E. eugeniae, utilisées dans le processus de vermicompostage sont issues des pays tropicaux (Temgoua et al., 2014). L'utilisation des espèces locales pour la transformation des matières organiques est un atout pour l'environnement (Dominguez \& GomezBrandon, 2012). Eudrilus eugeniae est également un atout majeur grâce à son utilisation dans la gestion des déchets urbains (Loongyai et al., 2011) et dans la détoxification des sols (Jones \& Lowe, 2012). Ce ver de terre permet de réduire de près de $30 \%$ les déchets organiques. Le vermicompostage ne génère pas d'odeur en principe (Chaoui, 2010). Aux Philipinnes, Adorada (2007) rapporte que le vermicompostage a permis de réduire les pollutions de l'air par la baisse du recours au feu pour la destruction des déchets organiques dans les fermes agricoles. C'est un apport non négligeable pour la préservation de l'environnement. Le potentiel de biodégradation des déchets organiques par E. eugeniae a été rapporté par Okwor et al. (2012) au Nigéria, où la production de déchets organiques est estimée à $0,45 \mathrm{~kg}$ par personne par jour. Au nord Vietnam, les effets positifs du vermicompostage sur l'environnement ont été rapportés par Doan et al. (2015), notamment par son impact sur la réduction de l'érosion, la baisse des eaux de ruissellement et la diminution de transfert de $\mathrm{NH}_{4}^{+}$et $\mathrm{NO}_{3}^{-}$dans l'eau.

\subsection{Importance agronomique}

Les vers de terre participent fortement à la fertilité des sols et à la croissance des plantes à travers les turricules qu'ils produisent (Hatti et al., 2012). Le passage de la matière organique à travers le tractus digestif du ver de terre améliore la composition microbienne et la teneur en élément minéraux du lombricompost (Lemtiri et al., 2014 ; Coulibaly et al., 2016). Le tableau 2 présente la composition du sol travaillé par le ver de terre et celle de ses déjections.
Table 2. Composition du sol vermoulu (WWS), déjection du ver de terre (EC) et sol témoin (BS) (d'après Tang et al., 2012 cité par Lemtiri et al., 2014). - Mineral element in worm-worked soil (WWS), earthworm casts (EC) and bulk soil (BS) (according to Tang et al., 2012 cited by Lemtiri et al., 2014).

\begin{tabular}{llll}
\hline Élément $\left(\mathrm{mg} \cdot \mathrm{kg}^{-1} \mathrm{sol} \mathrm{sec}\right)$ & WWS & EC & BS \\
\hline Azote & 2,3 & 3,8 & 3,4 \\
Phosphore & 1,1 & 1,4 & 1,2 \\
Potassium & 2,4 & 2,5 & 2,5 \\
Calcium & 3,7 & 5,0 & 3,9 \\
Magnésium & 1,1 & 1,4 & 1,2 \\
\hline
\end{tabular}

Eudrilus eugeniae est très utilisé pour le vermicompostage de différentes matières organiques en zone tropicale (Dominguez \& Edwards, 2010 ; Loongyai et al., 2011 ; Vijaya et al., 2012 ; Yesudhason et al., 2013), notamment en Afrique de l'Ouest (Coulibaly et al., 2011). C'est dans ce sens que Hatti et al. (2012) ont montré que l'abondance des macroéléments $(\mathrm{N}, \mathrm{P}, \mathrm{K}, \mathrm{S}, \mathrm{Ca}$, carbone organique) et des micro-éléments ( $\mathrm{Fe}, \mathrm{Mn}, \mathrm{Cu}$, et $\mathrm{Zn}$ ) dans les turricules d'E. eugeniae améliore la croissance et le rendement du haricot (Vigna mungo, Vigna radiata). La présence des métaux lourds dans le vermicompost utilisé pour fertiliser les terres de culture ne devrait pas poser de problème pour la plante et par la suite dans l'organisme humain (Han et al., 2008). En effet, le ver de terre est un accumulateur de métaux lourds présents dans le sol, il les rend alors moins disponibles pour les plantes (Coulibaly et al., 2016). Pour que la matière organique transformée soit utilisée de manière efficiente par la plante, il faudrait que celle-ci passe par un processus de stabilisation pour permettre que la quantité de métaux lourds absorbés par les végétaux soit infime et sans conséquence pour l'homme (Han Ko et al., 2008). Coulibaly et al. (2014) apportent une réponse à cette problématique en montrant qu'E. eugeniae a permis de stabiliser la matière organique du sol par vermicompostage en utilisant un mélange composé de 15 vers de terre par kg de déjections animales. C'est là l'un des avantages d'utiliser des vers de terre comme E. eugeniae dans le lombricompostage pour réduire la quantité des métaux lourds (Li et al., 2010). Il permet à la plante de disposer des nutriments $(\mathrm{N}, \mathrm{P}, \mathrm{K}, \mathrm{Ca})$ nécessaires à sa croissance sous une forme directement assimilable (Karthikairaj \& Isaiaasu, 2013). En plus de ces éléments nutritifs pour les cultures, Jesikha (2013) a mis en évidence la présence d'agents régulateurs de croissance des plantes dans le vermicompost utilisé pour amender les sols. Eudrilus eugeniae est également un atout majeur pour l'agriculture grâce à son action dans l'amélioration de la fertilité des terres 
cultivées (Jones \& Lowe, 2012). La lombriculture au sud Vietnam est devenue une activité prospère chez les paysans qui valorisent ainsi les vers de terre pour la fertilisation des terres agricoles (Francis et al., 2003). Cette pratique pourrait être étendue dans d'autres pays tropicaux où les problèmes de fertilité des sols et la gestion des déchets organiques se posent.

\subsection{Importance zootechnique}

La production des vers de terre pour l'alimentation animale suscite un intérêt particulier car le ver de terre est une source de protéine pour l'alimentation animale et humaine (Morin, 2004 ; Tiroesele \& Moreki, 2012). Certaines études ont montré l'intérêt de la lombriculture pour l'aviculture (Francis et al., 2003 ; Tiroesele et Moreki, 2012). Dans l'alimentation du poisson, la poudre de ver de terre a été utilisée en Malaisie (Abd Rahman Jabir et al., 2012), au Nigéria (Omoyinmi \& Olaoye, 2012), au Royaume Uni (Rawling et al., 2012) et pour l'alimentation du porc au Brésil (Vieira et al., 2004). En Thaïlande, E. eugeniae présente un intérêt nutritif de par son apport en protéine pour différents élevages de volailles et de poissons (Sivasankari et al., 2013). Ainsi, la poudre de ver de terre a pu être substituée à la protéine de poisson jusqu'à $25 \%$ dans la ration alimentaire de jeunes tilapias (Oreochromis niloticus), sans que cela n'ait entrainé une baisse de la croissance ni modifié la qualité de la chair du poisson (Abd Rahman Jabir et al., 2012). Cela fut confirmé par Omoyinmi \& Olaoye (2012) au Nigéria sur la croissance des poissons de la même espèce $(O$. niloticus $)$ nourris avec E. eugeniae. Des résultats similaires sur le gain de poids ont été obtenus avec le poisson chat hybride (o Heterobranchus longifilis X 0 Clarias gariepinus) appelé Heteroclarias nourri aussi avec de la poudre d'E. eugeniae (Monebi \& Ugwumba, 2013).

Différentes espèces de vers de terre, dont E. eugeniae, ont été utilisées pour évaluer leur apport en protéine pour l'aviculture, ce qui a permis de montrer que leur composition en protéine brute (E.foetida: $66,1 \%$, E. eugeniae : $58,4 \%$ et $P$. excavatus : 61,6\%) est proche des $61,0 \%$ de protéines brutes apportées par la farine de poisson (Reinecke et al., 1993). En général, le taux de protéine des vers de terre varie entre $64,5 \%$ et $72,9 \%$, suivant l'âge et l'espèce de ver de terre. Mais E.eugeniae reste une source de protéine intéressante pour l'alimentation animale avec un taux de 58,4 \% (Loongyai et al., 2011). La valeur nutritive d'E.eugeniae et son utilisation comme source de protéine dans l'alimentation des volailles ont été décrits dans plusieurs pays, notamment au Cameroun (Agbedé et al., 1994), au Vietnam (Francis et al., 2003), au Nigéria (Sogbesan et al., 2007), au Vénézuela (MorónFuenmayor et al., 2008), en Indonésie (Prayogi, 2011) et au Botswana (Chiripasi et al., 2013). Sogbesan et al.
(2007) ont montré que, bien que la farine de poisson ait une meilleure composition en protéine brute $(71,6 \%)$ par rapport à la poudre de ver de terre $(63,0 \%)$, l'utilisation de la protéine issue du ver de terre ne poserait aucun problème nutritionnel. Cela confirme bien le fait que l'utilisation de la poudre de ver de terre comme source de protéine, en lieu et place de la farine de poisson pour l'alimentation des volailles ne devrait pas avoir d'effets indésirables sur le gain de poids chez le poulet (Agbedé et al., 1994 ; Prayogi, 2011).

En plus des protéines, les vers de terre sont une source d'acides gras totaux $\left(6,6\right.$ à $\left.10,5 \mathrm{mg} \cdot \mathrm{g}^{-1}\right)$, de calcium $\left(1020\right.$ à $\left.7070 \mu \mathrm{g} \cdot \mathrm{g}^{1}\right)$, de fer $\left(1050\right.$ à $\left.2990 \mu \mathrm{g} \cdot \mathrm{g}^{-1}\right)$ et bien d'autres éléments nutritifs (Tableau 3) pour les animaux d'élevage (Tiroesele \& Moreki, 2012).

Tiroesele \& Moreki (2012) affirment que la similitude des acides aminés essentiels apportés par les vers de terre comme E. eugeniae et ceux du poisson (Tableau 4) permet d'envisager l'utilisation de la farine de vers de terre dans l'alimentation animale.

Table 3. Composition globale, minérale (\% matière sèche) et énergétique $\left(\mathrm{kJ} \cdot 100 \mathrm{~g}^{-1}\right)$ de la farine de ver de terre et de poisson (d'après Sogbesan \& Ugwamba, 2008 cité par Tiroesele \& Moreki, 2012) - Mineral (\% dry matter) and energetic $\left(\mathrm{kJ} \cdot 100 \mathrm{~g}^{-1}\right)$ composition of earthworm and fish meal (according to Sogbesan \& Ugwamba, 2008 cited by Tiroesele \& Moreki, 2012).

\begin{tabular}{lll}
\hline Nutriment & \multicolumn{2}{l}{ Protéine animale } \\
\cline { 2 - 3 } & $\begin{array}{l}\text { Farine de } \\
\text { ver de terre }\end{array}$ & $\begin{array}{l}\text { Farine de } \\
\text { poisson }\end{array}$ \\
\hline Protéine brute $(\%)$ & $63,3 \pm 4,5$ & $71,5 \pm 4,6$ \\
\hline Lipide $(\%)$ & $5,9 \pm 1,1$ & $8,0 \pm 1,4$ \\
\hline Fibre $(\%)$ & $1,9 \pm 0,2$ & $1,2 \pm 0,8$ \\
\hline Cendre $(\%)$ & $8,9 \pm 2,1$ & $7,3 \pm 1,2$ \\
\hline Extrait non azoté $(\%)$ & 11,8 & 3,2 \\
\hline Humidité $(\%)$ & 8,6 & 8,9 \\
\hline Énergie brute $\left(\mathrm{kj}^{\circ} \cdot 100 \mathrm{~g}^{-1}\right)$ & $1943,0 \pm 1,1$ & 2075,0 \\
\hline Rapport E/P & 32,0 & 29,0 \\
\hline Énergie métabolisable & 1476,0 & 1556,0 \\
\hline (kj·100 $\left.\mathrm{g}^{-1}\right)$ & & 1812,7 \\
\hline Énergie digestible $\left(\mathrm{kj}^{\prime} \cdot 100 \mathrm{~g}^{-1}\right)$ & 16,4 & $0,91 \pm 0,0$ \\
\hline Sodium $\left(\mathrm{g} \cdot 100 \mathrm{~g}^{-1}\right)$ & $0,43 \pm 0,0$ & $3,53 \pm 0,2$ \\
\hline Calcium $\left(\mathrm{g} \cdot 100 \mathrm{~g}^{-1}\right)$ & $0,53 \pm 0,1$ & $0,96 \pm 0,0$ \\
\hline Potassium $\left(\mathrm{g} \cdot 100 \mathrm{~g}^{-1}\right)$ & $0,62 \pm 0,0$ & $2,4 \pm 0,0$ \\
\hline Phosphore $\left(\mathrm{g} \cdot 100 \mathrm{~g}^{-1}\right)$ & $0,94 \pm 0,0$ & $0,08 \pm 0,0$ \\
\hline Magnésium $\left(\mathrm{g} \cdot 100 \mathrm{~g}^{-1}\right)$ & $/ /$ & \\
\hline
\end{tabular}


Table 4. Composition en acides aminés de deux sources de protéines $\left(\mathrm{g} \cdot 16 \mathrm{~g}^{-1} \mathrm{~N}\right)$ (d'après Reinecke et al., 1991 cité par Tiroesele \& Moreki, 2012) - Amino acids composition of two sources of proteins $\left(g \cdot 16 \mathrm{~g}^{-1} \mathrm{~N}\right)$ (according to Reinecke et al., 1991 cited by Tiroesele \& Moreki, 2012).

\begin{tabular}{llc}
\hline Acide aminé & Eudrilus eugeniae & Farine de poisson \\
\hline Thréonine & 4,3 & 3,8 \\
Sérine & 4,5 & 14,3 \\
Valine & 5,9 & 5,0 \\
Méthionine & 1,7 & 2,7 \\
Isoleucine & 4,6 & 4,1 \\
\hline Leucine & 9,6 & 7,8 \\
Tyrosine & 2,9 & 3,7 \\
Phénylalanine & 3,2 & 3,1 \\
Histidine & 3,1 & 2,2 \\
Lysine & 7,8 & 7,1 \\
Arginine & 9,2 & 4,6 \\
\hline
\end{tabular}

\section{FACTEURS AFFECTANT E. EUGENIAE}

Eudrilus eugeniae peut être influencé par les facteurs environnementaux tels que la température, le $\mathrm{pH}$, l'humidité et le type de sol (Morin, 2004). Eudrilus sp. peut vivre dans un milieu dont la température varie entre 15 et $30^{\circ} \mathrm{C}$, mais pour croitre et se reproduire normalement, le substrat doit avoir une température oscillant entre 25 et $28^{\circ} \mathrm{C}$ (Tahir \& Hamid, 2012). Une température inférieure à $15^{\circ} \mathrm{C}$ dans le milieu du ver de terre va entrainer une diminution de l'activité métabolique et de la croissance. Si la température est inférieure à $10^{\circ} \mathrm{C}$, E.eugeniae va réduire sa consommation alimentaire et cesser de se reproduire (Shagoti et al., 2001). Lorsque la température du milieu dépasse $30^{\circ} \mathrm{C}$, une baisse de la reproduction est observée et l'apparition du clitellum peut être retardée. Au-delà de $35^{\circ} \mathrm{C}$, le ver de terre meurt (Hallmann et al., 2014).

De façon générale, le ver de terre doit être maintenu dans un milieu dont le taux d'humidité varie entre 70 et $90 \%$, mais il croît et se reproduit mieux entre 70 et $80 \%$ (Shagoti et al., 2001). L'animal respire et détecte son environnement à travers la peau tant que le taux d'humidité est adéquat. Lorsque le sol est sec, le ver de terre est désorienté et il peut s'asphyxier très vite (Siegrist, 2011).

Le $\mathrm{pH}$ du sol dans lequel se trouve le ver de terre doit osciller entre 6,4 et 7,0. En deçà, il peut entrainer une réduction de la vitesse de croissance (Coulibaly \& Zoro Bi, 2010). Dans des conditions d'élevage expérimental, le $\mathrm{pH}$ du substrat a tendance à baisser. Pour le faire remonter, l'usage du carbonate de calcium est recommandé. Dans le cas où le pH est trop élevé, il faut le faire baisser par l'ajout d'un aliment acide tel que l'amidon (Shagoti et al., 2001). Toutefois, le ver de terre possède la capacité de modifier son environnement pour maintenir le $\mathrm{pH}$ entre 6,5 et 7 (WormsRus, 2014 ; Coulibaly et al., 2016).

Eudrilus eugeniae possède des cellules photosensibles sur la peau. Ces cellules créent un stimulus douloureux et paralysant au contact de la lumière et cela peut lui être fatal. Pour se protéger, il sécrète un mucus protecteur sur sa peau. C'est pourquoi le ver de terre préfère rester dans l'obscurité (Morin, 1999 ; Chaoui, 2010).

Les vers de terre ne tolèrent pas une forte concentration d'ammoniac. De ce fait, Eudrilus sp. ne survit pas dans des fientes ou des bouses fraiches (Morin, 2004). Le sel est fatal pour le ver de terre : la salinité a un effet destructeur sur sa peau. Lorsqu'elle dépasse $5 \mathrm{mg} \cdot \mathrm{g}^{-1}$, les vers de terre tendent à s'échapper du milieu (Chaoui, 2010).

Eudrilus eugeniae est absent des sols non adaptés à son développement, comme les déserts et les régions glaciales. L'absence de matière organique dont se nourrit le ver de terre influence la répartition géographique de ce lombric (Ansari \& Saywack, 2010). En se nourrissant de différents types de matières organiques, le ver de terre absorbe parfois des substances toxiques ou des micro-organismes pathogènes présents dans le sol. Eudrilus eugeniae peut ainsi devenir une source de contamination pour d'autres animaux consommateurs de ver de terre (Wang et al., 2009). Le ver de terre est reconnu pour être un accumulateur des métaux lourds (Guérin et al., 2011 ; Bystrzejewska-Piotrowska et al., 2012) et un hôte intermédiaire de certains parasites des volailles, dont il ingère les œufs (Munroe, 2006). La consommation d'une matière organique contenant des substances médicamenteuses, telles que des vermifuges rémanents présents dans les fumiers, peut affecter le ver de terre, ce qui explique que l'utilisation de ces fumiers doit être contrôlée (Shagoti et al., 2001)

Les insecticides néonicotinoïdes utilisés en agriculture, comme l'imidaclopride, peuvent se retrouver dans les sols. Ils sont nocifs pour les vers de terre (Sherwan, 2013). En milieu agricole, l'usage intensif des pesticides présente ainsi des risques de toxicité pour les Oligochètes en général et E. eugeniae en particulier, et entraine la disparition des vers de terre dans certaines zones agricoles du fait de cette utilisation intensive (Jones \& Lowe, 2012).

\section{CONCLUSIONS ET PERSPECTIVES}

Cette synthèse bibliographique visait à regrouper les connaissances sur le ver de terre E. eugeniae. Elle révèle l'importance de l'élaboration d'une clé d'identification 
spécifique à l'espèce, à l'instar de celles élaborées pour d'autres groupes de vers de terre, ce qui faciliterait encore le processus d'identification de ce ver de terre. Les informations recueillies ne permettent que d'avoir une connaissance relativement superficielle de la biologie de cet animal.

Les études consacrées à E. eugeniae ces dernières années montrent l'intérêt grandissant de ce ver de terre pour la science. Mais les données collectées révèlent aussi un déficit d'informations sur E. eugeniae dans la région équatoriale et notamment au Gabon. L'exploitation de cette source non conventionnelle de protéine implique le développement de la lombriculture, principalement au Gabon où ce ver de terre peut jouer un rôle majeur dans la gestion des déchets organiques, l'alimentation animale et la fertilisation des terres agricoles.

L'élevage d'E.eugeniae n'ayant jamais été fait au Gabon,l'approfondissement des études sur cette espèce, dans cette région, devrait permettre de fournir des informations nouvelles sur sa description, sa biologie, son écologie, son comportement et son importance économique. Ces données pourraient contribuer à améliorer les connaissances existantes sur E. eugeniae.

\section{Bibliographie}

Abd Rahman Jabir M.D., Razak S.A. \& Vikineswary S., 2012. Nutritive potential and utilization of super worm (Zophobas morio) meal in the diet of Nile tilapia (Oreochromis niloticus) juvenile. Afr. J. Biotechnol., 11(24), 6592-6598.

Adorada J.L., 2007. Assessment of vermicomposting as a waste management technology and a livelihood alternative in the Philippines. J. Environ. Sci. Manage., 10, 28-39.

Agdebe G., Nguekam \& Mpoame M., 1994. Essai d'utilisation de la farine de vers de terre Eudrilus eugeniae dans l'alimentation des poulets de chair en finition. Tropicultura, 12(1), 3-5.

Ansari A.A. \& Saywack P., 2010. Taxonomical studies on some earthworm species in Guyana. World J. Zool., 5, 162-166.

Ansari A.A. \& Saywack P., 2011. Identification and classification of earthworm species in Guyana. Int. J. Zool.Res., 7, 93-99.

Brown G.G. et al., 2013. Terrestrial oligochaete research in Latin America: the importance of the Latin American meetings on oligochaete ecology and taxonomy. Appl. Soil Ecol., 69, 2-12.

Bruyeer R. \& Simus P., 2012. Le calcul du rapport carbone azote, http://users.swing.be/compost/Main_Rapport\%20 _C_N.htm, (23/10/2013).

Butt K.R. \& Grigoropoulou N., 2010. Basic research tools for earthworm ecology. Appl. Environ. Soil Sci., 2010, ID 562816.
Bystrzejewska-Piotrowska G., Asztemborska M., Giska I. \& Mikoszewski A., 2012. Influence of earthworms on extractability of metals from soils contaminated with $\mathrm{Al}_{2} \mathrm{O}_{3}, \mathrm{TiO}_{2}, \mathrm{Zn}$, and $\mathrm{ZnO}$ nanoparticles and microparticles of $\mathrm{Al}_{2} \mathrm{O}_{3}$. Pol. J. Environ. Stud., 21, 313-319.

Chaoui H.,2010. Vermicompostage (ou lombricompostage) : le traitement des déchets organiques par les vers de terre. In : Ministère de l'Agriculture, de l'Alimentation et des Affaires rurales, éd. Fiche technique $n^{\circ} 10$. Guelph, ON, Canada : Ministère de l'Agriculture, de l'Alimentation et des Affaires rurales.

Chiripasi S.C., Moreki J.C., Nsoso S.J. \& Letso M., 2013. Effect of feeding mopane worm meal on mineral intake, retention and utilization in guinea fowl under intensive system. Int. J. Poultry Sci., 12(1), 19-28.

Cluzeau D., 2013. Déterminer les vers de terre, https:// ecobiosoil.univ-rennes1.fr/e107_files/downloads/ OPVT_Determiner_les_Vers_de_Terre.pdf, (20/10/2016).

Coulibaly S.S. \& Zoro Bi I.A., 2010. Influence of animal wastes on growth and reproduction of the African earthworm species Eudrilus eugeniae (Oligochaeta). Eur. J. Soil Biol., 46, 225-229.

Coulibaly S.S., Kouassi K.I., Tondoh E.J. \& Zoro Bi I.A., 2011. Impact of the population size of the earthworm Eudrilus eugeniae (Kinberg) on the stabilization of animal wastes during vermicomposting. Philippine Agric. Sci., 94, 359-367.

Coulibaly S.S. et al., 2014. Influence of the population size of the earthworm Eudrilus eugeniae on the heavy metal content reduction during vermicomposting of animal wastes. Appl. Sci.Rep., 7(2), 96-103.

Coulibaly S.S. et al., 2016. Vermicomposts improve yields and seeds quality of Lagenaria siceraria in Côte d'Ivoire. Int. J. Agron. Agric. Res., 8, 26-37.

Crow W.T., 2012. Earthworm, suborder Crassiclitellata, cohort Terrimegradili (Jamieson, 1988), http://edis.ifas. ufl.edu/pdffiles/IN/IN94600.pdf, (25/03/2014).

Deka H. et al., 2011. Vermicomposting of distillation waste of citronella plant (Cymbopogon winterianus Jowitt.) employing Eudrilus eugeniae. Bioresour. Technol., 102, 6944-6950.

Doan T.T. et al., 2015. Impact of compost, vermicompost and biochar on soil fertility, maize yield and soil erosion in Northern Vietnam: a three year mesocosm experiment. Sci. Total Environ., 514, 147-154.

Dominguez J. \& Edwards C.A., 2010. Biology and ecology of earthworm species used for vermicomposting. In: Edwards C.A., Arancon N.Q. \& Sherman R.L., eds. Vermitechnology: earthworms, organic wastes and environmental management. Boca Raton, Fl, USA: CRC Press, 27-38.

Domínguez J. \& Gómez-Brandón M., 2010. Ciclos de vida de las lombrices de tierra aptas para el vermicompostaje (Life cycles of vermicomposting earthworms). Acta Zool.Mex., 26, 1-8. 
FAO, 2004. Ressources alimentaires. Rome : FAO, 16-28.

Fernández R. et al., 2011. Ultrastructural and molecular insights into three populations of Aporrectodea trapezoides (Dugés, 1828) (Oligochaeta, Lumbricidae) with different reproductive modes. Pedobiologia, 54, 281-290.

Francis F. et al., 2003. Technique de lombriculture au sud Vietnam. Biotechnol. Agron. Soc. Environ., 7, 171-175.

Gomez-Brandon M., Lores M. \& Dominguez J., 2012. Species-specific effects of epigeic earthworms on microbial community structure during first stages of decomposition of organic matter. Plos One, 7, 1-8.

Guérin J.L., Balloy D. \& Villate D., 2011. Maladies des volailles. Paris : Éditions France Agricole.

Hallmann C.A. et al., 2014. Declines in insectivorous birds are associated with high neonicotinoid concentrations Nature, 511, 341-343.

Han Ko J. et al., 2008. Evaluation of maturity parameters and heavy metal contents in composts made from animal manure. Waste Manage., 28, 813-820.

Hatti S.S. et al., 2012. Effet of Eudrilus eugeniae vermiwash on the growth of plants. J. Experiment. Zool., 15, 63-67.

James S.W. \& Divina G.B., 2012. Earthworms (Clitellata: Acanthodrilidae, Almidae, Eudrilidae, Glossoscolecidae, Ocnerodrilidae) of the coastal region of Gamba, OgoouéMaritime Province, southwestern Gabon. Zootaxa, $\mathbf{3 4 5 8}$, 133-148.

Jesikha M., 2013. Growth of medicinal and economical plants in vermicompost for sustainable development. Res. J. Anim. Vet. Fish. Sci., 1(3), 1-6.

Jesikha M., 2015. Effect of paper waste on the life cycle of earthworm Eudrilus eugeniae (Kinberg). SIRJ-BES, 1, 7-12.

Jones D.T. \& Lowe C.N., 2012. Key to common British earthworms of amenity grassland, http://www. opalexplorenature.org/sites/default/files/7/file/OPALgrassland-earthworm-key.pdf, (12/11/2014).

Karmakar S., Brahmachari K., Gangopadhyay A. \& Choudhury S.R., 2012. Recycling of different available organic wastes through vermicomposting. E-J.Chem., 9, 801-806.

Karthikairaj K. \& Isaiarasu L., 2013. Effect of vermiwash on the growth of mulberry cuttings. World J.Agric. Sci., 9(1), 69-72.

Kooch Y. \& Jalilvand H., 2008. Earthworms as ecosystem engineers and the most important detritivors in forest soils. Pak. J. Biol. Sci., 11, 819-825.

Lemtiri A. et al., 2014. Impacts of earthworms on soil components and dynamics. A review. Biotechnol. Agron. Soc. Environ., 18, 121-133.

Li L., Xu Z., Wu J. \& Tian G., 2010. Bioaccumulation of heavy metals in the earthworms Eisenia fetida in relation to bioavailable metal concentrations in pig manure. Bioresour. Technol., 101, 3430-3436.

Loongyai W., Bangrak P. \& Chantsavang S., 2011. External morphological comparison, taxonomic revision and molecular differentiation of the four economically important species of earthworm in Thailand. Int. J. Agric. Biol., 13, 553-558.

Lowe C.N. \& Butt K.R., 2005. Earthworm culture, maintenance and species selection in chronic ecotoxicological studies: a critical review. Eur. J. Soil Biol., 43, 281-288.

Misra R.V., Roy R.N. \& Hiraoka H., 2005. Méthodes de compostage au niveau de l'exploitation agricole. Rome : FAO.

Monebi C.O. \& Ugwumba A.A.A., 2013. Utilization of the earthworm, Eudrilus eugeniae in the diet of Heteroclarias fingerlings. Int. J. Fish. Aquacult., 5(2), 19-25.

Moraes M.J.d., Filho D.O., Martins J.H. \& Santos L.C., 2012. Electric signals for separation of earthworms (Eudrilus eugeniae). R. Bras. Eng. Agríc. Ambiental, 16, 1137-1142.

Morin R., 1999. Exploitation et élevage des vers de terre pour le marché des appâts vivants. In: Ministère de l'Agriculture, des Pêcheries et de l'Alimentation, éd. Document d'information DADD-20. Québec, Canada : Ministère de l'Agriculture, des Pêcheries et de l'Alimentation.

Morin E., 2004. Lombricompostage, une façon écologique de traiter les résidus organiques. In : Eco-quartier PeterMcGill P., éd. Guide pratique. Montréal, Canada : Ministère de l'Environnement du Québec.

Morón-Fuenmayor O.E. et al., 2008. Effect of earthworm (Eisenia spp.) meal inclusion on dressing and physicalchemical characteristics of quail meat (Coturnix coturnix japonica). Rev. Fac. Agron. (LUZ), 25, 674-685.

Munroe G., 2006. Guide du lombricompostage et de lombriculture à la ferme. Québec, Canada: Centre d'agriculture biologique du Canada.

Nayak S.K. \& Sahu S.K., 2013. Vermicomposting of poultry litter using Eudrilus eugeniae. Ecoscan, 3, 267-271.

Oboh B.O., Akintobi D.O. \& Ejidereonwu C., 2007. Morphometric studies in Eudrilus eugeniae populations from different locations in Lagos, Nigeria. Nat. Sci., 5, 16-21.

Okwor A.C., Ebenebe C.I. \& Anizoba M.A., 2012. Biodegradation of domestic organic waste using earthworm (Eudrilus eugeniae): a veritable tool for agricultural and environmental sustainability. Int. J. Agric. Biosci., 1, 39-41.

Omoyinmi G.A.K. \& Olaoye O.J., 2012. Growth performance of nile tilapia-Oreochromis niloticus fed diets containing different sources of animal protein. Libyan Agric. Res. Center J. Int., 3, 18-23.

Parthasarathi K., 2007. Life cycle of Lampito moretii (Kinberg) in comparaison with Eudrilus eugeniae (Kinberg) cultured on different substrates. J. Environ. Biol., 28, 803-812.

Pérès G. et al., 2011. Earthworm indicators as tools for soil monitoring, characterization and risk assessment. 
An example from the national bioindicator programme (France). Pedobiologia, 54, 77-87.

Prayogi H.S., 2011. The effect of earthworm meal supplementation in the diet on quail's growth performance in attempt to replace the usage of fish meal. Int. J. Poult. Sci., 10(10), 804-806.

Rajendran P., Jayakumar E., Sripathi Kandula \& Gunasekaran P., 2008. Vermiculture and vermicomposting biotechnology for organic farming and rural economic development, http://www.eco-web.com/ edi/080211.html, (6/11/2016).

Rawling M.D. et al., 2012. Haemato-immunological and growth response of mirror carp (Cyprinus carpio) fed a tropical earthworm meal in experimental diets. Fish Shellfish Immunol., 32, 1002-1007.

Reinecke A.J., Viljoen S.A. \& Gayman R.J., 1992. The suitability of Eudrilus eugeniae, Perionyx excavatus and Ersenia fetida (Oligochaeta) for vermicomposting in southern Africa in terms of their temperature requirements. Soil Biol. Biochem., 24, 1295-1307.

Reinecke A.J. \& Viljoen S.A., 1993. Cocoon deposition by the African nightcrawler, Eudrilus eugeniae, (Oligachaeta). Pedobiologia, 37, 351-356.

Schuch R., Pelzek A.J., Kan S. \& Fischetti V.A., 2010. Prevalence of Bacillus anthracis-like organisms and bacteriophages in the intestinal tract of the earthworm Eisenia fetida. Appl. Environ. Microbiol., 76, 2286-2294.

Shagoti U.M., Amoji S.D., Biradar V.A. \& Biradar P.M., 2001. Effect of temperature on growth and reproduction of the epigeic earthworm, Eudrilus eugeniae (Kinberg). J. Environ. Biol., 22, 213-217.

Sherwan T.A., 2013. The impact of four pesticides on the earthworm Lumbricus terrestris (Annelida; Oligochaeta). Int. J. Curr. Res. Rev., 5, 1-5.

Siegrist M., 2011. Le lombric sort de l'ombre, http://www. terrenature.ch/jardin/16062011-1214-le-lombric-sortde-lombre, (15/03/2014).

Sivasankari B., Indumathi S. \& Anandharaj M., 2013. A study on life cycle of earthworm Eudrilus eugeniae. Int . J. Res. Pharm. Life Sci., 1, 64-67.
Sogbesan A.O., Ugwamba A.A.A. \& Madu C.T., 2007. Productivity potentials and nutritional values of semiarid zone earthworm (Hyperiodrilus euryaulos; Clausen, 1967) cultured in organic wastes as fish meal supplement. Pak. J. Biol. Sci., 10, 2992-2997.

Tahir T.A. \& Hamid F.S., 2012. Vermicomposting of two types of coconut wastes employing Eudrilus eugeniae: a comparative study. Int. J. Recycl. Org. Waste Agric., 1, 1-6.

Temgoua E., Ngnikam E., Dameni H. \& Kouedeu Kameni G.S., 2014. Valorisation des ordures ménagères par compostage dans la ville de Dschang, Cameroun. Tropicultura, 32(1), 28-36.

Tiroesele B. \& Moreki J.C., 2012. Termites and earthworms as potential alternative sources of protein for poultry. Int . J. Agron. Vet. Med. Sci., 6(5), 368-376.

Vieira M.L., Ferreira A.S. \& Donzelle J.L., 2004. Digestibilidade da farinha de minhoca para suínos. Bol. Ind. Anim. N. Odessa, 61(1), 83-89.

Vijaya T.M. et al., 2012. Morphological and histological studies on the vermicomposting Indian earthworm Eudrilus eugeniae. World J. Zool., 7, 165-170.

Wang Q. et al., 2009. Indication of soil heavy metal pollution with earthworms and soil microbial biomass carbon in the vicinity of an abandoned copper mine in Eastern Nanjing, China. Eur. J. Soil Biol., 45, 229-234.

WormsRus, 2014. About earthworms, http://www.wormsrus. co.nz/aboutearthworms.html, (2/11/2016).

Yadav A. \& Garg V.K., 2011. Recycling of organic wastes by employing Eisenia foetida. Bioresour. Technol., 102, 2874-2880.

Yesudhason B.V. et al., 2013. Unique phenotypes in the sperm of the earthworm Eudrilus eugeniae for assessing radiation hazards. Environ. Monit. Assess., 185, 47454752.

Zirbes L. et al., 2011. Earthworms use odor cues to locate and feed on microorganisms in soil. PLoS One, 6, 219227.

(74 réf.) 